\title{
Article \\ On Partial Sums of Analytic Univalent Functions
}

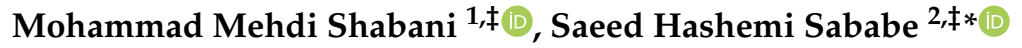 \\ 1 Department of Mathematics, University of Shahrood, Shahrood, Iran.; \\ Mohammadmehdishabani@ymail.com \\ 2 Young Researchers and Elite Club, Malard Branch, Islamic Azad University, Malard, Iran.; \\ Hashemi_1365@yahoo.com \\ $\ddagger$ These authors contributed equally to this work.
}

Received: date; Accepted: date; Published: date

\begin{abstract}
Partial sums of analytic univalent functions and partial sums of starlike have been investigated extensively by several researchers. In this paper, we investigate a partial sums of convex harmonic functions that are univalent and sense preserving in the open unit disk.
\end{abstract}

Keywords: Harmonic; Univalent, Convex; Partial sums

MSC: Primary 30C45; Secondary 30C50

\section{Introduction}

A continuous function $f=u+i v$ is a complex valued harmonic function in a complex domain $\Omega \subset \mathbb{C}$ if both $u$ and $v$ are real harmonic in $\Omega$.

In any simply connected domain $\Omega \subset \mathbb{C}$, we may write $f=h+\bar{g}$, where $h$ and $g$ are analytic in $\Omega$. We call $h$ the analytic part and $g$ the co-analytic part of $f$. A necessary and sufficient condition for $f$ to be locally univalent and sense-preserving in $\Omega$ is that $\left|h^{\prime}(z)\right|>\left|g^{\prime}(z)\right|$ in $\Omega$. (See [2]).

Denote by $\mathcal{S}_{\mathcal{H}}$ the class of functions $f=h+\bar{g}$ that are harmonic univalent and sense-preserving in $\mathbb{D}=\{z \in \mathbb{C}:|z|<1\}$ for which $f(0)=f_{z}(0)-1=0$. Then for $f=h+\bar{g} \in \mathcal{S}_{\mathcal{H}}$, the analytic functions $h$ and $g$ can be expressed as

$$
h(z)=z+\sum_{k=2}^{\infty} a_{k} z^{k}, \quad g(z)=\sum_{k=1}^{\infty} b_{k} z^{k}, \quad\left|b_{1}\right|<1 .
$$

A function $f$ of the form (1) is harmonic convex of order $\alpha, 0 \leq \alpha<1$, denoted by $K_{H}(\alpha)$, if it satisfies

$$
\frac{\partial}{\partial \theta}\left\{\arg \left(\frac{\partial}{\partial \theta} f\left(r e^{i \theta}\right)\right\}=\operatorname{Re}\left\{\frac{z\left(z h^{\prime}(z)\right)^{\prime}+\overline{z\left(z g^{\prime}(z)\right)^{\prime}}}{z h^{\prime}(z)-\overline{z g^{\prime}(z)}}\right\} \geq \alpha,\right.
$$

where $0 \leq \theta \leq 2 \pi,|z|=r<1$.

As shown recently by Jahangiri [7] a sufficient condition for a function of the form (1) to be in $K_{H}(\alpha)$ is that

$$
\sum_{k=1}^{\infty}\left(\frac{k(k-\alpha)}{1-\alpha}\left|a_{k}\right|+\frac{k(k+\alpha)}{1-\alpha}\left|b_{k}\right|\right) \leq 2
$$

In 1985, Silvia[13] studied the partial sums of convex functions of order $\alpha$. Later, Silverman [12], Abubaker and Darus[1], Dixit and Porwal[4], Frasin[5,6], Raina and Bansal[10], Rosy et al.[11] and Porwal and Dixit[9] exhibited some results on partial sums for various classes of analytic functions. 
Here, we investigate a partial sums of convex harmonic functions.

Now, we let the sequences of partial sums of functions of the form (1) with $b_{1}=0$, have forms

$$
\begin{aligned}
& f_{m}(z)=z+\sum_{k=2}^{m} a_{k} z^{k}+\sum_{k=2}^{\infty} \overline{b_{k} z^{k}} \\
& f_{n}(z)=z+\sum_{k=2}^{\infty} a_{k} z^{k}+\sum_{k=2}^{n} \overline{b_{k} z^{k}} \\
& f_{m, n}(z)=z+\sum_{k=2}^{m} a_{k} z^{k}+\sum_{k=2}^{n} \overline{b_{k} z^{k}}
\end{aligned}
$$

and study some some bounds for them.

\section{Main Results}

The main aims of this paper is the following theorems which determine sharp lower bounds for some classes of univalent functions. In the following theorem, we try it for $\operatorname{Re}\left\{f(z) / f_{m}(z)\right\}$. If $f(z)$ of the form (1) with $b_{1}=0$ satisfies condition (2), then

$$
\operatorname{Re}\left\{\frac{f(z)}{f_{m}(z)}\right\} \geq \frac{m(m+2-\alpha)}{(m+1)(m+1-\alpha)}, \quad z \in \mathbb{D} .
$$

The result (3) is sharp with the function

$$
f(z)=z+\frac{1-\alpha}{(m+1)(m+1-\alpha)} z^{m+1}
$$

Proof. By setting

$$
\begin{aligned}
A_{1}(z)=\sum_{k=2}^{m} r^{k-1} e^{i(k-1) \theta} a_{k} & +\sum_{k=2}^{\infty} r^{k-1} e^{-i(k+1) \theta} \overline{b_{k}} \\
& +\frac{(m+1)(m+1-\alpha)}{1-\alpha}\left[\sum_{k=m+1}^{\infty} r^{k-1} e^{i(k-1) \theta} a_{k}\right],
\end{aligned}
$$

and

$$
B_{1}(z)=\sum_{k=2}^{m} r^{k-1} e^{i(k-1) \theta} a_{k}+\sum_{k=2}^{\infty} r^{k-1} e^{-i(k+1) \theta} \overline{b_{k}}
$$

We have

$$
\frac{(m+1)(m+1-\alpha)}{1-\alpha}\left[\frac{f(z)}{f_{m}(z)}-\frac{m(m+2-\alpha)}{(m+1)(m+1-\alpha)}\right]=\frac{1+A_{1}(z)}{1+B_{1}(z)} .
$$

Let $\frac{1+A_{1}(z)}{1+B_{1}(z)}=\frac{1+\omega(z)}{1-\omega(z)}$, then $\omega(z)=\frac{A_{1}+(z)-B_{1}(z)}{2+A_{1}(z)+B_{1}(z)}$. It is easy to see that

$$
|\omega(z)| \leq \frac{\frac{(m+1)(m+1-\alpha)}{1-\alpha}\left(\sum_{k=m+1}^{\infty}\left|a_{k}\right|\right)}{2-2\left(\sum_{k=2}^{m}\left|a_{k}\right|+\sum_{k=2}^{\infty}\left|b_{k}\right|\right)-\frac{(m+1)(m+1-\alpha)}{1-\alpha}\left(\sum_{k=m+1}^{\infty}\left|a_{k}\right|\right)} .
$$


We claim that the right hand side of the above inequality is bounded above by 1 , if and only if

$$
\sum_{k=2}^{m}\left|a_{k}\right|+\sum_{k=2}^{\infty}\left|b_{k}\right|+\frac{(m+1)(m+1-\alpha)}{1-\alpha}\left(\sum_{k=m+1}^{\infty}\left|a_{k}\right|\right) \leq 1,
$$

It suffices to show that the L. H. S. of (5) is bounded above by

$$
\sum_{k=1}^{\infty}\left(\frac{k(k-\alpha)}{1-\alpha}\left|a_{k}\right|+\frac{k(k+\alpha)}{1-\alpha}\left|b_{k}\right|\right)
$$

which is equivalent to

$$
\begin{aligned}
\sum_{k=2}^{m}\left(\frac{k(k-\alpha)}{1-\alpha}-1\right)\left|a_{k}\right| & +\sum_{k=2}^{\infty}\left(\frac{k(k+\alpha)}{1-\alpha}-1\right)\left|b_{k}\right| \\
& +\sum_{k=m+1}^{\infty}\left(\frac{k(k-\alpha)}{1-\alpha}-\frac{(m+1)(m+1-\alpha)}{1-\alpha}\right)\left|a_{k}\right| \geq 0
\end{aligned}
$$

To complete the proof we show that $f(z)=z+\frac{1-\alpha}{(m+1)(m+1-\alpha)} z^{m+1}$ gives the sharp result. Let $z=r e^{i \frac{\pi}{m}}$ we have

$$
\begin{aligned}
\frac{f(z)}{f_{m}(z)}=1 & +\frac{1-\alpha}{(m+1)(m+1-\alpha)} z^{m} \\
& \Longrightarrow 1-\frac{1-\alpha}{(m+1)(m+1-\alpha)}=\frac{m(m+2-\alpha)}{(m+1)(m+1-\alpha)}
\end{aligned}
$$

when $r \rightarrow 1^{-}$.

In next theorem, we try it on the inverse of the fraction. If $f(z)$ of the form (1) with $b_{1}=0$ satisfies condition (2), then

$$
\operatorname{Re}\left\{\frac{f_{m}(z)}{f(z)}\right\} \geq \frac{(m+1)(m+1-\alpha)}{m(m+2-\alpha)+2(1-\alpha)}, \quad z \in \mathbb{D} .
$$

The result (6) is sharp with the function given by (4).

Proof. Let

$$
\begin{aligned}
A_{2}(z)=\sum_{k=2}^{m} r^{k-1} e^{i(k-1) \theta} a_{k} & +\sum_{k=2}^{\infty} r^{k-1} e^{-i(k+1) \theta} \overline{b_{k}} \\
& -\frac{(m+1)(m+1-\alpha)}{1-\alpha}\left(\sum_{k=m+1}^{\infty} r^{k-1} e^{i(k-1) \theta} a_{k}\right),
\end{aligned}
$$

and $B_{2}(z)$ be as the $B_{1}(z)$ on the previews proof. We may write

$$
\begin{aligned}
& \frac{1+\omega(z)}{1-\omega(z)}=\frac{m(m+2-\alpha)+2(1-\alpha)}{1-\alpha}\left[\frac{f_{m}(z)}{f(z)}-\frac{(m+1)(m+1-\alpha)}{m(m+2-\alpha)+2(1-\alpha)}\right] \\
& =\frac{1+A_{2}(z)}{1+B_{2}(z)}
\end{aligned}
$$


where

$$
|\omega(z)| \leq \frac{\frac{m(m+2-\alpha)+2(1-\alpha)}{1-\alpha}\left[\sum_{k=m+1}^{\infty}\left|a_{k}\right|\right]}{2-2\left(\sum_{k=2}^{m}\left|a_{k}\right|+\sum_{k=2}^{\infty}\left|b_{k}\right|\right)-\frac{m(m+2-\alpha)}{1-\alpha}\left(\sum_{k=m+1}^{\infty}\left|a_{k}\right|\right)} \leq 1
$$

Equivalently

$$
\sum_{k=2}^{m}\left|a_{k}\right|+\sum_{k=2}^{\infty}\left|b_{k}\right|+\frac{m(m+2-\alpha)+(1-\alpha)}{1-\alpha}\left(\sum_{k=m+1}^{\infty}\left|a_{k}\right|\right) \leq 1
$$

since the left hand side of (7) is bounded above by $\sum_{k=1}^{\infty}\left(\frac{k(k-\alpha)}{1-\alpha}\left|a_{k}\right|+\frac{k(k+\alpha)}{1-\alpha}\left|b_{k}\right|\right)$, the proof is complete.

As two corollaries, we are interesting to know about these type of inequalities on derivatives of $f$. If $f(z)$ of the form (1) with $b_{1}=0$ satisfies condition (2), then

$$
\operatorname{Re}\left\{\frac{f^{\prime}(z)}{f_{m}^{\prime}(z)}\right\} \geq \frac{m}{m+1-\alpha}, \quad z \in \mathbb{D} .
$$

The result (8) is sharp with the function given by (4).

Proof. Let

$$
\begin{aligned}
A_{3}=\sum_{k=2}^{m} k r^{k-1} e^{i(k-1) \theta} a_{k} & -\sum_{k=2}^{\infty} k r^{k-1} e^{-i(k+1) \theta} \overline{b_{k}} \\
& +\frac{m+1-\alpha}{1-\alpha}\left[\sum_{k=m+1}^{\infty} k r^{k-1} e^{i(k-1) \theta} a_{k}\right]
\end{aligned}
$$

and

$$
B_{3}=\sum_{k=2}^{m} k r^{k-1} e^{i(k-1) \theta} a_{k}-\sum_{k=2}^{\infty} k r^{k-1} e^{-i(k+1) \theta} \overline{b_{k}}
$$

We have

$$
\frac{1+\omega(z)}{1-\omega(z)}=\frac{m+1-\alpha}{1-\alpha}\left[\frac{f^{\prime}(z)}{f_{m}^{\prime}(z)}-\frac{m}{m+1-\alpha}\right]=\frac{1+A_{3}}{1+B_{3}}
$$

Then

$$
\omega(z)=\frac{\frac{m+1-\alpha}{1-\alpha}\left[\sum_{k=m+1}^{\infty} k r^{k-1} e^{i(k-1) \theta} a_{k}\right]}{2+2 A_{3}} .
$$

Similarlly to the Theorem 2 .

If $f(z)$ of the form (1) with $b_{1}=0$ satisfies condition (2), then

$$
\operatorname{Re}\left\{\frac{f_{m}^{\prime}(z)}{f^{\prime}(z)}\right\} \geq \frac{m+1-\alpha}{m+2(1-\alpha)}, \quad z \in \mathbb{D} .
$$

The result (9) is sharp with the function given by (4). 
Proof. Since

$$
\frac{1+\omega(z)}{1-\omega(z)}=\frac{m+2(1-\alpha)}{1-\alpha}\left[\frac{f_{m}^{\prime}(z)}{f^{\prime}(z)}-\frac{m+1-\alpha}{m+2(1-\alpha)}\right]
$$

in a simillar way of the proof of Corollary 2, we obtain the results.

In the following, we prove the same theorems for $f_{n}(z)$. If $f(z)$ of the form (1) with $b_{1}=0$ satisfies condition (2), then

$$
\operatorname{Re}\left\{\frac{f(z)}{f_{n}(z)}\right\} \geq \frac{n(n+2+\alpha)}{(n+1)(n+1+\alpha)}, \quad z \in \mathbb{D}
$$

The result (10) is sharp with the function

$$
f(z)=z+\frac{1-\alpha}{(n+1)(n+1+\alpha)} \bar{z}^{n+1} .
$$

Proof. Let

$$
\begin{aligned}
A_{5}=\sum_{k=2}^{\infty} r^{k-1} e^{i(k-1) \theta} a_{k} & +\sum_{k=2}^{n} r^{k-1} e^{-i(k+1) \theta} \overline{b_{k}} \\
& +\frac{(n+1)(n+1+\alpha)}{1-\alpha}\left[\sum_{k=n+1}^{\infty} r^{k-1} e^{-i(k+1) \theta} \overline{b_{k}}\right],
\end{aligned}
$$

and

$$
B_{5}=\sum_{k=2}^{\infty} r^{k-1} e^{i(k-1) \theta} a_{k}+\sum_{k=2}^{n} r^{k-1} e^{-i(k+1) \theta} \overline{b_{k}}
$$

We have

$$
\frac{1+\omega(z)}{1-\omega(z)}=\frac{(n+1)(n+1+\alpha)}{1-\alpha}\left[\frac{f(z)}{f_{n}(z)}-\frac{n(n+2+\alpha)}{(n+1)(n+1+\alpha)}\right]=\frac{1+A_{5}}{1+B_{5}}
$$

where

$$
\omega(z)=\frac{\frac{(n+1)(n+1+\alpha)}{1-\alpha}\left[\sum_{k=n+1}^{\infty} r^{k-1} e^{-i(k+1) \theta} \overline{b_{k}}\right]}{2+2 A_{5}}
$$

Then

$$
|\omega(z)| \leq \frac{\frac{(n+1)(n+1+\alpha)}{1-\alpha}\left[\sum_{k=n+1}^{\infty}\left|b_{k}\right|\right]}{2-2\left(\sum_{k=2}^{\infty}\left|a_{k}\right|+\sum_{k=2}^{n}\left|b_{k}\right|\right)-\frac{(n+1)(n+1+\alpha)}{1-\alpha}\left(\sum_{k=n+1}^{\infty}\left|b_{k}\right|\right)}
$$

This last expression is bounded above by 1 , if and only if

$$
\sum_{k=2}^{\infty}\left|a_{k}\right|+\sum_{k=2}^{n}\left|b_{k}\right|+\frac{(n+1)(n+1+\alpha)}{1-\alpha}\left(\sum_{k=n+1}^{\infty}\left|b_{k}\right|\right) \leq 1
$$


It suffices to show that the left hand side of (12) is bounded above by

$$
\sum_{k=1}^{\infty}\left(\frac{k(k-\alpha)}{1-\alpha}\left|a_{k}\right|+\frac{k(k+\alpha)}{1-\alpha}\left|b_{k}\right|\right)
$$

which is equivalent to

$$
\begin{aligned}
\sum_{k=2}^{\infty}\left(\frac{k(k-\alpha)}{1-\alpha}-1\right)\left|a_{k}\right| & +\sum_{k=2}^{n}\left(\frac{k(k+\alpha)}{1-\alpha}-1\right)\left|b_{k}\right| \\
& +\sum_{k=n+1}^{\infty}\left(\frac{k(k+\alpha)}{1-\alpha}-\frac{(n+1)(n+1+\alpha)}{1-\alpha}\right)\left|b_{k}\right| \geq 0
\end{aligned}
$$

To complete the proof, we see that $f(z)=z+\frac{1-\alpha}{(n+1)(n+1+\alpha)} \bar{z}^{n+1}$ gives the sharp result. Let $z=r e^{i \frac{\pi}{n+2}}$ we have

$$
\begin{aligned}
\frac{f(z)}{f_{n}(z)}=1 & +\frac{1-\alpha}{(n+1)(n+1+\alpha)} r^{n} e^{-\frac{i \pi}{n+2}(n+2)} \\
& \Longrightarrow 1-\frac{1-\alpha}{(n+1)(n+1+\alpha)}=\frac{n(n+2+\alpha)}{(n+1)(n+1+\alpha)}
\end{aligned}
$$

when $r \rightarrow 1^{-}$. This completes the proof.

If $f(z)$ of the form (1) with $b_{1}=0$ satisfies condition (2), then

$$
\operatorname{Re}\left\{\frac{f_{n}(z)}{f(z)}\right\} \geq \frac{(n+1)(n+1+\alpha)}{n(n+2+\alpha)+2}, \quad z \in \mathbb{D}
$$

The result (13) is sharp with the function given by (11).

Proof. Let

$$
\begin{aligned}
A_{6}=\sum_{k=2}^{\infty} r^{k-1} e^{i(k-1) \theta} a_{k} & +\sum_{k=2}^{n} r^{k-1} e^{-i(k+1) \theta} \overline{b_{k}} \\
& -\frac{(n+1)(n+1+\alpha)}{1-\alpha}\left[\sum_{k=n+1}^{\infty} r^{k-1} e^{-i(k+1) \theta} \overline{b_{k}}\right],
\end{aligned}
$$

and

$$
B_{6}=\sum_{k=2}^{\infty} r^{k-1} e^{i(k-1) \theta} a_{k}+\sum_{k=2}^{\infty} r^{k-1} e^{-i(k+1) \theta} \overline{b_{k}}
$$

It is easy to see that

$$
\frac{1+\omega(z)}{1-\omega(z)}=\frac{n(n+2+\alpha)+2}{1-\alpha}\left[\frac{f_{n}(z)}{f(z)}-\frac{(n+1)(n+1+\alpha)}{n(n+2+\alpha)+2}\right]=\frac{1+A_{6}}{1+B_{6}}
$$

Details of the proof is omitted and is simillar to the Theorem 2.

If $f(z)$ of the form (1) with $b_{1}=0$ satisfies condition (2),

(i) If $n(n+2+\alpha)+2 \alpha \geq m(m+2-\alpha)$ or for all $k \geq 2$ we have $b_{k}=0$ then

$$
\operatorname{Re}\left\{\frac{f(z)}{f_{m, n}(z)}\right\} \geq \frac{m(m+2-\alpha)}{(m+1)(m+1-\alpha)}, \quad z \in \mathbb{D}
$$


(ii) if $n(n+2+\alpha)+2 \alpha \leq m(m+2-\alpha)$ or for all $k \geq 2$ we have $a_{k}=0$ then

$$
\operatorname{Re}\left\{\frac{f(z)}{f_{m, n}(z)}\right\} \geq \frac{n(n+2+\alpha)}{(n+1)(n+1+\alpha)}, \quad z \in \mathbb{D} \text {. }
$$

Proof. (i) Let

$$
\begin{aligned}
A_{7}= & +\sum_{k=2}^{m} r^{k-1} e^{i(k-1) \theta} a_{k}+\sum_{k=2}^{n} r^{k-1} e^{-i(k+1) \theta} \overline{b_{k}} \\
& +\frac{(m+1)(m+1-\alpha)}{1-\alpha}\left[\sum_{k=m+1}^{\infty} r^{k-1} e^{i(k-1) \theta} a_{k}+\sum_{k=n+1}^{\infty} r^{k-1} e^{-i(k+1) \theta} \overline{b_{k}}\right],
\end{aligned}
$$

and

$$
B_{7}=\sum_{k=2}^{m} r^{k-1} e^{i(k-1) \theta} a_{k}+\sum_{k=2}^{n} r^{k-1} e^{-i(k+1) \theta} \overline{b_{k}}
$$

We have

$$
\frac{1+\omega(z)}{1-\omega(z)}=\frac{(m+1)(m+1-\alpha)}{1-\alpha}\left[\frac{f(z)}{f_{m, n}(z)}-\frac{m(m+2-\alpha)}{(m+1)(m+1-\alpha)}\right]=\frac{1+A_{7}}{1+B_{7}}
$$

Then

$$
\omega(z)=\frac{\frac{(m+1)(m+1-\alpha)}{1-\alpha}\left[\sum_{k=m+1}^{\infty} r^{k-1} e^{i(k-1) \theta} a_{k}+\sum_{k=n+1}^{\infty} r^{k-1} e^{-i(k+1) \theta} \overline{b_{k}}\right]}{2+2 A_{7}} .
$$

Therefore

$$
|\omega(z)| \leq \frac{\frac{(m+1)(m+1-\alpha)}{1-\alpha}\left[\sum_{k=m+1}^{\infty}\left|a_{k}\right|+\sum_{k=n+1}^{\infty}\left|b_{k}\right|\right]}{2-2\left(\sum_{k=2}^{m}\left|a_{k}\right|+\sum_{k=2}^{n}\left|b_{k}\right|\right)-\frac{(m+1)(m+1-\alpha)}{1-\alpha}\left(\sum_{k=m+1}^{\infty}\left|a_{k}\right|+\sum_{k=n+1}^{\infty}\left|b_{k}\right|\right)} .
$$

This last expression is bounded above by 1 , if and only if

$$
\sum_{k=2}^{m}\left|a_{k}\right|+\sum_{k=2}^{n}\left|b_{k}\right|+\frac{(m+1)(m+1-\alpha)}{1-\alpha}\left(\sum_{k=m+1}^{\infty}\left|a_{k}\right|+\sum_{k=n+1}^{\infty}\left|b_{k}\right|\right) \leq 1 .
$$

Since the left hand side of (14) is bounded above by

$$
\sum_{k=1}^{\infty}\left(\frac{k(k-\alpha)}{1-\alpha}\left|a_{k}\right|+\frac{k(k+\alpha)}{1-\alpha}\left|b_{k}\right|\right)
$$

the following inequality holds

$$
\begin{aligned}
& \sum_{k=2}^{m}\left(\frac{k(k-\alpha)}{1-\alpha}-1\right)\left|a_{k}\right|+\sum_{k=m+1}^{\infty}\left(\frac{k(k-\alpha)}{1-\alpha}-\frac{(m+1)(m+1-\alpha)}{1-\alpha}\right)\left|a_{k}\right| \\
& +\sum_{k=2}^{n}\left(\frac{k(k+\alpha)}{1-\alpha}-1\right)\left|b_{k}\right|+\sum_{k=n+1}^{\infty}\left(\frac{k(k+\alpha)}{1-\alpha}-\frac{(m+1)(m+1-\alpha)}{1-\alpha}\right)\left|b_{k}\right| \geq 0 .
\end{aligned}
$$


To see $f(z)=z+\frac{1-\alpha}{(m+1)(m+1-\alpha)} z^{m+1}$ gives the sharp result, let $z=r e^{i \frac{\pi}{m}}$. We have

$$
\begin{aligned}
\frac{f(z)}{f_{m, n}(z)}=1 & +\frac{1-\alpha}{(m+1)(m+1-\alpha)} z^{m} \\
& \Longrightarrow 1-\frac{1-\alpha}{(m+1)(m+1-\alpha)}=\frac{m(m+2-\alpha)}{(m+1)(m+1-\alpha)}
\end{aligned}
$$

when $r \rightarrow 1^{-}$.

(ii) Let

$$
\begin{aligned}
& C_{7}=\sum_{k=2}^{m} r^{k-1} e^{i(k-1) \theta} a_{k}+\sum_{k=2}^{n} r^{k-1} e^{-i(k+1) \theta} \overline{b_{k}} \\
& +\frac{(n+1)(n+1+\alpha)}{1-\alpha}\left[\sum_{k=m+1}^{\infty} r^{k-1} e^{i(k-1) \theta} a_{k}+\sum_{k=n+1}^{\infty} r^{k-1} e^{-i(k+1) \theta} \overline{b_{k}}\right],
\end{aligned}
$$

and

$$
D_{7}=\sum_{k=2}^{m} r^{k-1} e^{i(k-1) \theta} a_{k}+\sum_{k=2}^{n} r^{k-1} e^{-i(k+1) \theta} \overline{b_{k}}
$$

We have

$$
\frac{1+\omega(z)}{1-\omega(z)}=\frac{(n+1)(n+1+\alpha)}{1-\alpha}\left[\frac{f(z)}{f_{m, n}(z)}-\frac{n(n+2+\alpha)}{(n+1)(n+1+\alpha)}\right]=\frac{1+C_{7}}{1+D_{7}} .
$$

Then

$$
\omega(z)=\frac{\frac{(n+1)(n+1+\alpha)}{1-\alpha}\left[\sum_{k=m+1}^{\infty} r^{k-1} e^{i(k-1) \theta} a_{k}+\sum_{k=n+1}^{\infty} r^{k-1} e^{-i(k+1) \theta} \overline{b_{k}}\right]}{2+2 C_{7}} .
$$

\section{Consequently}

$$
|\omega(z)| \leq \frac{\frac{(n+1)(n+1+\alpha)}{1-\alpha}\left[\sum_{k=m+1}^{\infty}\left|a_{k}\right|+\sum_{k=n+1}^{\infty}\left|b_{k}\right|\right]}{2-2\left(\sum_{k=2}^{m}\left|a_{k}\right|+\sum_{k=2}^{n}\left|b_{k}\right|\right)-\frac{(n+1)(n+1+\alpha)}{1-\alpha}\left(\sum_{k=m+1}^{\infty}\left|a_{k}\right|+\sum_{k=n+1}^{\infty}\left|b_{k}\right|\right)} .
$$

This last expression is bounded above by 1 , if and only if

$$
\sum_{k=2}^{m}\left|a_{k}\right|+\sum_{k=2}^{n}\left|b_{k}\right|+\frac{(n+1)(n+1+\alpha)}{1-\alpha}\left(\sum_{k=m+1}^{\infty}\left|a_{k}\right|+\sum_{k=n+1}^{\infty}\left|b_{k}\right|\right) \leq 1 .
$$

It suffices to show that the left hand side of (15) is bounded above by

$$
\sum_{k=1}^{\infty}\left(\frac{k(k-\alpha)}{1-\alpha}\left|a_{k}\right|+\frac{k(k+\alpha)}{1-\alpha}\left|b_{k}\right|\right)
$$


which is equivalent to

$$
\begin{aligned}
& \sum_{k=2}^{m}\left(\frac{k(k-\alpha)}{1-\alpha}-1\right)\left|a_{k}\right|+\sum_{k=m+1}^{\infty}\left(\frac{k(k-\alpha)}{1-\alpha}-\frac{(n+1)(n+1+\alpha)}{1-\alpha}\right)\left|a_{k}\right| \\
& +\sum_{k=2}^{n}\left(\frac{k(k+\alpha)}{1-\alpha}-1\right)\left|b_{k}\right|+\sum_{k=n+1}^{\infty}\left(\frac{k(k+\alpha)}{1-\alpha}-\frac{(n+1)(n+1+\alpha)}{1-\alpha}\right)\left|b_{k}\right| \geq 0 .
\end{aligned}
$$

To show that $f(z)=z+\frac{1-\alpha}{(n+1)(n+1+\alpha)} \bar{z}^{n+1}$ gives the sharp result, let $z=r e^{i \frac{\pi}{n+2}}$. We have

$$
\begin{aligned}
\frac{f(z)}{f_{m, n}(z)}=1 & +\frac{1-\alpha}{(n+1)(n+1+\alpha)} r^{n} e^{-\frac{i \pi}{n+2}(n+2)} \\
& \Longrightarrow 1-\frac{1-\alpha}{(n+1)(n+1+\alpha)}=\frac{n(n+2+\alpha)}{(n+1)(n+1+\alpha)},
\end{aligned}
$$

when $r \rightarrow 1^{-}$. The result follows.

If $f(z)$ of the form (1) with $b_{1}=0$ satisfies condition (2),

(i) if $n(n+2+\alpha)+2 \alpha \geq m(m+2-\alpha)$ or for all $k \geq 2$ we have $b_{k}=0$ then

$$
\operatorname{Re}\left\{\frac{f_{m, n}(z)}{f(z)}\right\} \geq \frac{(m+1)(m+1-\alpha)}{m(m+2-\alpha)+2(1-\alpha)}, \quad z \in \mathbb{D} .
$$

(ii) if $n(n+2+\alpha)+2 \alpha \leq m(m+2-\alpha)$ or for all $k \geq 2$ we have $a_{k}=0$ then

$$
\operatorname{Re}\left\{\frac{f_{m, n}(z)}{f(z)}\right\} \geq \frac{(n+1)(n+1+\alpha)}{n(n+2+\alpha)+2}, \quad z \in \mathbb{D} .
$$

Proof. (i) Let

$$
\begin{aligned}
A_{8} & =\sum_{k=2}^{m} r^{k-1} e^{i(k-1) \theta} a_{k}+\sum_{k=2}^{n} r^{k-1} e^{-i(k+1) \theta} \overline{b_{k}} \\
& -\frac{(m+1)(m+1-\alpha)}{1-\alpha}\left[\sum_{k=m+1}^{\infty} r^{k-1} e^{i(k-1) \theta} a_{k}+\sum_{k=n+1}^{\infty} r^{k-1} e^{-i(k+1) \theta} \overline{b_{k}}\right],
\end{aligned}
$$

and

$$
B_{8}=\sum_{k=2}^{\infty} r^{k-1} e^{i(k-1) \theta} a_{k}+\sum_{k=2}^{\infty} r^{k-1} e^{-i(k+1) \theta} \overline{b_{k}}
$$

We have

$$
\frac{1+\omega(z)}{1-\omega(z)}=\frac{m(m+2-\alpha)+2(1-\alpha)}{1-\alpha}\left[\frac{f_{m, n}(z)}{f(z)}-\frac{(m+1)(m+1-\alpha)}{m(m+2-\alpha)+2(1-\alpha)}\right]=\frac{1+A_{8}}{1+B_{8}} .
$$

Then

$$
|\omega(z)| \leq \frac{\frac{m(m+2-\alpha)+2(1-\alpha)}{1-\alpha}\left[\sum_{k=m+1}^{\infty}\left|a_{k}\right|+\sum_{k=n+1}^{\infty}\left|b_{k}\right|\right]}{2-2\left(\sum_{k=2}^{m}\left|a_{k}\right|+\sum_{k=2}^{n}\left|b_{k}\right|\right)-\frac{m(m+2-\alpha)}{1-\alpha}\left(\sum_{k=m+1}^{\infty}\left|a_{k}\right|+\sum_{k=n+1}^{\infty}\left|b_{k}\right|\right)} \leq 1 .
$$


Therefore

$$
\sum_{k=2}^{m}\left|a_{k}\right|+\sum_{k=2}^{n}\left|b_{k}\right|+\frac{m(m+2-\alpha)+(1-\alpha)}{1-\alpha}\left(\sum_{k=m+1}^{\infty}\left|a_{k}\right|+\sum_{k=n+1}^{\infty}\left|b_{k}\right|\right) \leq 1
$$

Since the left hand side of (16) is bounded above by

$$
\sum_{k=1}^{\infty}\left(\frac{k(k-\alpha)}{1-\alpha}\left|a_{k}\right|+\frac{k(k+\alpha)}{1-\alpha}\left|b_{k}\right|\right)
$$

the proof is complete.

(ii) Let

$$
\begin{aligned}
C_{8}= & \sum_{k=2}^{m} r^{k-1} e^{i(k-1) \theta} a_{k}+\sum_{k=2}^{n} r^{k-1} e^{-i(k+1) \theta} \overline{b_{k}} \\
& -\frac{(n+1)(n+1+\alpha)}{1-\alpha}\left[\sum_{k=m+1}^{\infty} r^{k-1} e^{i(k-1) \theta} a_{k}+\sum_{k=n+1}^{\infty} r^{k-1} e^{-i(k+1) \theta} \overline{b_{k}}\right]
\end{aligned}
$$

and

$$
D_{8}=\sum_{k=2}^{\infty} r^{k-1} e^{i(k-1) \theta} a_{k}+\sum_{k=2}^{\infty} r^{k-1} e^{-i(k+1) \theta} \overline{b_{k}}
$$

We have

$$
\frac{1+\omega(z)}{1-\omega(z)}=\frac{n(n+2+\alpha)+2}{1-\alpha}\left[\frac{f_{m, n}(z)}{f(z)}-\frac{(n+1)(n+1+\alpha)}{n(n+2+\alpha)+2}\right]=\frac{1+C_{8}}{1+D_{8}} .
$$

Then

$$
|\omega(z)| \leq \frac{\frac{n(n+2+\alpha)+2}{1-\alpha}\left[\sum_{k=m+1}^{\infty}\left|a_{k}\right|+\sum_{k=n+1}^{\infty}\left|b_{k}\right|\right]}{2-2\left(\sum_{k=2}^{m}\left|a_{k}\right|+\sum_{k=2}^{n}\left|b_{k}\right|\right)-\frac{n(n+2+\alpha)+2 \alpha}{1-\alpha}\left(\sum_{k=m+1}^{\infty}\left|a_{k}\right|+\sum_{k=n+1}^{\infty}\left|b_{k}\right|\right)} \leq 1 .
$$

Therefore

$$
\sum_{k=2}^{m}\left|a_{k}\right|+\sum_{k=2}^{n}\left|b_{k}\right|+\frac{n(n+2+\alpha)+(1+\alpha)}{1-\alpha}\left(\sum_{k=m+1}^{\infty}\left|a_{k}\right|+\sum_{k=n+1}^{\infty}\left|b_{k}\right|\right) \leq 1 .
$$

Since the left hand side of (17) is bounded above by

$$
\sum_{k=1}^{\infty}\left(\frac{k(k-\alpha)}{1-\alpha}\left|a_{k}\right|+\frac{k(k+\alpha)}{1-\alpha}\left|b_{k}\right|\right)
$$

which completes the proof.

If $f(z)$ of the form (1) with $b_{1}=0$ satisfies condition (2), then

$$
\operatorname{Re}\left\{\frac{f^{\prime}(z)}{f_{m, n}^{\prime}(z)}\right\} \geq \frac{m}{m+1-\alpha}, \quad \text { for } n>m, z \in \mathbb{D} .
$$

The result (18) is sharp with the function given by (4). 
Proof. Let

$$
\begin{aligned}
A_{9}= & \sum_{k=2}^{m} k r^{k-1} e^{i(k-1) \theta} a_{k}-\sum_{k=2}^{n} k r^{k-1} e^{-i(k+1) \theta} \overline{b_{k}} \\
& +\frac{m+1-\alpha}{1-\alpha}\left[\sum_{k=m+1}^{\infty} k r^{k-1} e^{i(k-1) \theta} a_{k}-\sum_{k=n+1}^{\infty} k r^{k-1} e^{-i(k+1) \theta} \overline{b_{k}}\right]
\end{aligned}
$$

and

$$
B_{9}=\sum_{k=2}^{m} k r^{k-1} e^{i(k-1) \theta} a_{k}-\sum_{k=2}^{n} k r^{k-1} e^{-i(k+1) \theta} \overline{b_{k}}
$$

We have

$$
\frac{1+\omega(z)}{1-\omega(z)}=\frac{m+1-\alpha}{1-\alpha}\left[\frac{f^{\prime}(z)}{f_{m, n}^{\prime}(z)}-\frac{m}{m+1-\alpha}\right]=\frac{1+A_{9}}{1+B_{9}}
$$

Then

$$
\omega(z)=\frac{\frac{m+1-\alpha}{1-\alpha}\left[\sum_{k=m+1}^{\infty} k r^{k-1} e^{i(k-1) \theta} a_{k}-\sum_{k=n+1}^{\infty} k r^{k-1} e^{-i(k+1) \theta} \overline{b_{k}}\right]}{2+2 A_{9}}
$$

and

$$
|\omega(z)| \leq \frac{\frac{m+1-\alpha}{1-\alpha}\left[\sum_{k=m+1}^{\infty} k\left|a_{k}\right|-\sum_{k=n+1}^{\infty} k\left|b_{k}\right|\right]}{2-2\left(\sum_{k=2}^{m} k\left|a_{k}\right|+\sum_{k=2}^{n} k\left|b_{k}\right|\right)-\frac{m+1-\alpha}{1-\alpha}\left(\sum_{k=m+1}^{\infty} k\left|a_{k}\right|+\sum_{k=n+1}^{\infty} k\left|b_{k}\right|\right)} \leq 1 .
$$

Therefore

$$
\sum_{k=2}^{m} k\left|a_{k}\right|+\sum_{k=2}^{n} k\left|b_{k}\right|+\frac{m+1-\alpha}{1-\alpha}\left(\sum_{k=m+1}^{\infty} k\left|a_{k}\right|+\sum_{k=n+1}^{\infty} k\left|b_{k}\right|\right) \leq 1
$$

Since the left hand side of (19) is bounded above by

$$
\sum_{k=1}^{\infty}\left(\frac{k(k-\alpha)}{1-\alpha}\left|a_{k}\right|+\frac{k(k+\alpha)}{1-\alpha}\left|b_{k}\right|\right)
$$

the proof is complete.

In a similar way to the previews theorem, we may prove the next corollary. If $\mathrm{f}$ of the form (1) with $b_{1}=0$ satisfies condition $(2)$, then

$$
\operatorname{Re}\left\{\frac{f_{m, n}^{\prime}(z)}{f^{\prime}(z)}\right\} \geq \frac{m+1-\alpha}{m+2(1-\alpha)}, \quad z \in \mathbb{D} .
$$

The result $(20)$ is sharp with the function $f(z)=z+\frac{1-\alpha}{(m+1)(m+1-\alpha)} z^{m+1}$.

\section{Acknowledgments}

The first Author would like to express his thanks to his colleague, professor Ali Ebadian, for his suggestions. This work is partially supported the first author by a grant of The University of 
Shahrood and the second author by a grant of Young Researchers and Elite Club, Malard Branch, Islamic Azad University.

\section{References}

1. A. Abubaker, M. Darus, Partial sums of analytic functions involving generalized ChoKwon-Srivastava operator, Int. J. Open Prob. Compl. Anal., 2010, 2(3), pp. 181-188.

2. J. Clunie, T. Sheil-Small, Harmonic univalent functions, Ann. Acad. Sci. Fenn. Ser. A I Math., 1984, 9, pp. 3-25.

3. K.K. Dziok, J. Jahangiri, H. Silverman, Harmonic functions with varying coecients, J. Inequal. Appl., 2016, 139 , pp. 1-12.

4. K.K. Dixit, S. Porwal, A convolution approach on partial sums of certain analytic and univalent functions, J. Inequal. Pure Appl. Math., 2009, 10(4), pp. 1-17.

5. B.A. Frasin, Partial sums of certain analytic and univalent functions, Acta Math. Acad. Paed. Nyir., 2005, 21, pp. 135-145.

6. B.A. Frasin, Generalization of partial sums of certain analytic and univalent functions, Applied Mathematics Letters, 2008, 21(7), pp. 735-741.

7. J.M. Jahangiri, Coefficient bounds and univalence criteria for harmonic functionswith negative coefficients, Ann. Univ. Mariae Cruie-Sklodowska Sect. A, 1998, 52(2), pp. 57-66.

8. J. Jahangiri, H. Silverman, E.M. Silvia, Construction of planar harmonic functions, Int. J. Math. Math. Sci., 2007, Art. ID 70192, pp. 1-11.

9. S. Porwal, K.K. Dixit, Partial sums of starlike harmonic univalent functions, Kyungpook Mathematical Journal, 2010, 50(3), pp. 433-445.

10. R.K. Raina, D. Bansal, Some properties of a new class of analytic functions defined in terms of a Hadamard product, J. Inequal. Pure. Appl. Math., 2008, 9(1), pp. 1-9.

11. T. Rosy, K.G. Subramanian, G. Murugusundaramoorthy, Neighbourhoods and partial sums of starlike functions based on Ruscheweyh derivatives, J.Ineq. Pure and Appl. Math., 2003, 4(64), pp. 1-8.

12. H. Silverman, Partial sums of starlike and convex functions, Journal of Mathematical Analysis and Applications, 1997, 209(1), pp. 221-227.

13. E.M. Silvia, Partial sums of convex functions of order $\alpha$, Houston.J.Math., Math.Soc., 1985, 11(3), pp. $397-404$. 